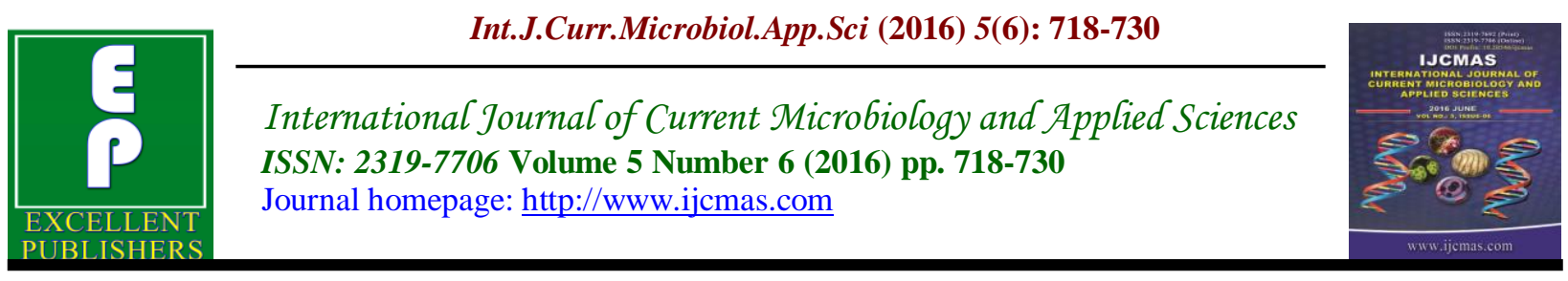

Original Research Article

http://dx.doi.org/10.20546/ijcmas.2016.506.078

\title{
Isolation and Qualitative Selection of Fungi for Production of Lignocellulolytic Enzymes
}

\author{
Ajit Kaur $^{1 *}$ and Urmila Gupta Phutela ${ }^{2}$ \\ ${ }^{1}$ Department of Microbiology, College of Basic Sciences and Humanities, Punjab Agricultural \\ University, Ludhiana-141004, Punjab, India \\ ${ }^{2}$ School of Energy Studies for Agriculture, College of Agricultural Engineering and Technology, \\ Punjab Agricultural University, Ludhiana-141004, Punjab, India \\ *Corresponding author
}

\begin{tabular}{|c|c|}
\hline & A B S T R A C T \\
\hline & \multirow{11}{*}{$\begin{array}{l}\text { Microorganisms especially Fungi are well known for their capability to excrete } \\
\text { industrially important enzymes into the environment. In this purposed study, thirty } \\
\text { four mesophilic as well as thermophilic fungal species were isolated from different } \\
\text { sources like, decaying wood, decaying kinnow fruit, compost and soil. The } \\
\text { isolated species were then purified and qualitatively selected for their cellulolytic, } \\
\text { lignolytic and hemicellulose degrading potential using agar plate assay method. } \\
\text { Carboxy Methyl Cellulose was used as substrate for cellulase activity, xylan was } \\
\text { used as a substrate for xylanase activity; Remazol brilliant blue (RBB) dye and } \\
\text { guaiacol were used as substrate to evaluate lignolytic activity in the isolated fungi. } \\
\text { A total of } 15 \text { fungal isolates and the standard cultures namely Phanerochaete } \\
\text { chrysosporium MTCC 787, Trichoderma reesei MTCC 164, T. harzianum MTCC } \\
792 \text {, Coriolus versicolor MTCC } 138, \quad \text { Pleurotus ostreatus MTCC 142, } \\
\text { Aspergillus oryzae NCIM 1212, Penicillium roquefortii NCIM 712, Thermoascus } \\
\text { aurantiacus MTCC } 375, \text { Humicola fuscoatra MTCC } 1409 \text { showed redness zone } \\
\text { on guaiacol. Two mesophilic isolates namely A } 4 \text { and A5; two thermophilic isolates } \\
\text { A25 and A31 are the potential cellulose, hemicelluloses and lignin degraders which } \\
\text { can further be used for enhancing biogas production. Among standard cultures P. } \\
\text { chrysosporium and T. aurantiacus produced remarkable amounts of } \\
\text { lignocellulolytic enzymes. }\end{array}$} \\
\hline & \\
\hline & \\
\hline Mesophilic and & \\
\hline $\begin{array}{l}\text { fungi, Isolation, } \\
\text { Screening. }\end{array}$ & \\
\hline & \\
\hline Arti & \\
\hline & \\
\hline & \\
\hline & \\
\hline & \\
\hline
\end{tabular}

\section{Introduction}

Lignocellulose describes the three major constituents of biomass, namely cellulose, hemicelluloses and lignin. Cellulose is the major chemical component of the fiber wall of plant, which is a homopolysaccharide composed entirely of D-glucose linked to each other by $\beta$-1,4-glycosidic bonds by degree of polymerization ranging from 1,000 in bleached kraft pulps to 10,000 in native wood. Cellulose is degraded by the enzymes which belong predominantly to hydrolases, cleaving the glycosidic bonds (Schmidt, 2006). Hemicelluloses are complex heterogeneous polysaccharides made up of different monomeric residues, 
such as D-xylose, D-glucose, D-arabinose, D-mannose and D-glucuronic acid. They are classified according to the monomeric sugar in the backbone of the polymer, e.g. mannan $(\beta-1,4-$ linked mannose) or xylan $(\beta-1,4-$ linked xylose) hemicelluloses. The third main constituent is lignin, which is a complex macromolecule formed by the dehydrogenative polymerization of three phenyl propane units namely p-coumaryl alcohol, coniferyl alcohol and sinapyl alcohol joined through ether bonds (Hofrichter, 2002).

Among the sources of these enzymes microbial sources are preferred industrially because of the short generation times of the microbes and so large volumes of enzymes can be obtained within a short time. Microorganisms such as bacteria and fungi are capable of degrading these complex polymers. Among these microorganisms, filamentous fungi appear to be the most efficient degraders (Jørgensen, 2003, Hakala et al.,2006; Jiménez-Tobon et al., 2003; Kamitsuji et al., 2004; Lankinen et al., 2005; Nuske et al., 2002; Palma et al., 2000; Petruccioli et al.,2009; Steffen et al.,2002; Susla et al.,2008; Silva et al.,2008; Sklenar et al.,2010; Singh et al.,2011; Taboada-Puig et al.,2011; Wang et al.,2008). These fungi include species from the ascomycetes (e.g. T. reesei), basidiomycetes including white-rot fungi (e.g. P. chrysosporium), brown-rot fungi (e.g. Fomitopsis palustris) and finally a few anaerobic species (e.g. Orpinomyces sp.) (Ljungdahl, 2008; Yoon et al., 2007; Dashtban et al., 2009). Lignocellulose degradation by these fungi is performed by complex mixtures of cellulases (Dashtban et al.,2009; Weng et al., 2008) hemicellulases (Yoon et al.,2007) and ligninases (Weng et al., 2008; Sanchez, 2009) reflecting the complexity of the materials. Nagendran et al., (2009) cited that, a multitude of enzymatic activities are required for the conversion of lignocellulosic biomass into useful (fermentable) products. Known essential activities to degrade crystalline cellulose include cellobiohydrolase $(\mathrm{CBH})$, endo- $\beta 1,4$-glucanase (EG), and $\beta$ glucosidase (BG). A larger suite of enzymes is necessary to depolymerize hemicelluloses, including endo- $\beta 1,4$-xylanase (EX), $\beta$ xylosidase $(\mathrm{BX}), \quad \alpha$-arabinosidase, $\alpha$ glucuronidase, and esterase. The ability to produce specific enzymes for degradation of different carbon and nitrogen sources are due to the diverse habitat where they are found (Prathumpai, 2003). Over the last few years, scientific investigations on such industrial enzyme production from microorganisms have intensified, mainly because of the world wide interest in exploiting renewable resources of biomass as a source of chemicals and liquid fuels. There has been a growing interest in studying the lignin-modifying enzymes of a wider array of white rot fungi for use in various biotechnological applications.

For the year 2010, demands for thermostable, strong, as well as highly specific industrial enzymes in the global market were valued at $\$ 3.6$ billion; it was estimated that the value will grow at a compounded annual growth rate (CAGR) of $9.1 \%$ and reach $\$ 6$ billion by 2016 (Dewan, 2012). Around $75 \%$ of these enzymes of the global enzyme market are lignocellulases. But, one of the bottlenecks of this strategy is the high preparation cost of pure commercial enzymes, and relatively low conversion efficiency, which make it less practicable for a vast industrial scale (Kang et al., 2004). For this, many innovative efforts like genetic modification, microbiological selection, enzymes immobilization and protein engineering have been made to innovate an alternative tuning tool to enhance the features of the enzymes and 
their production process (Barbosa et al., 2013). For this, the recombinant production and regulation of lignolytic enzymes has also been intensively studied in filamentous fungi (Conesa et al.,2000; Irie et al.,2001; Li et al.,2001), some yeasts (Jiang et al.,2008a), some novel strains of bacteria (Whitwam \& Tien ,1996; Bharagava et al.,2009; Mishra \& Thakur 2010; Yadav et al.,2011) and alson in some insect (Johnson et al.,1992; Pease et al.,1991) hosts with successful production but modest yields of active enzyme. Filamentous fungi secrete these enzymes into the medium and have higher activities in contrast to yeasts and bacteria (Krisana et al., 2005). Therefore, the present work reports the isolation and primary screening of lignocelluloses degrading fungi.

\section{Materials and Methods}

\section{Isolation and Purification of Lignocellulolytic Fungi}

Different fungal cultures were isolated from different sources like decaying wood, decaying kinnow fruit, soil, compost, digested slurry and plant debris. One gram of sample was vortexed with $99 \mathrm{ml}$ of sterilized distilled water to make uniform suspension. Heavy particles were allowed to settle and clear supernatant was used for serial dilution. One $\mathrm{ml}$ of serially diluted sample was pour plated on potato dextrose agar medium (PDA), each containing chloramphenicol $(50 \mathrm{mg} / \mathrm{l})$ and incubated at $30 \pm 2^{\circ} \mathrm{C}$ for mesophilic and $45 \pm 2^{\circ} \mathrm{C}$ for thermophilic cultures. Further, the isolated colonies were sub cultured again on fresh agar plates to purify the cultures.

\section{Screening of Lignocellulolytic Fungi}

The agar plate assay method (Okino et al., 2000) also recommended by International
Union of Pure and Applied Chemistry (IUPAC) was used in the investigation for the qualitative selection of the isolated and purified cultures for their lignocellulose degradation potential. Fungal species were grouped as high and low celluloytic isolates on the basis of cellulase activity using Index of Relative Enzyme Activity (ICMC). Cellulolytic fungi grown on paddy straw agar (PSA) medium supplemented with Carboxy methyl cellulose were evaluated after 7 days for the production of cellulolytic enzymes by flooding with $0.15 \% \mathrm{I}_{2}$ solution. The diameter of clear zone around the fungal colony, gave an approximate indication of cellulase activities. Hemicellulose degrading fungi were anylzed by supplementing the paddy straw agar (PSA) medium with xylan and flooding the colonies with $1 \%$ congo red. The clearance zone around the fungal colony indicates xylanase activities. Remazol brilliant blue (RBB) and guaiacol were used as indicator dyes for lignin degradation and potency index was calculated by the following formula:

Potency index $=$ size of clearance zone $\left(\mathrm{cm}^{2}\right) /$ size of colony $\left(\mathrm{cm}^{2}\right)$

The concentration of RBB used was $0.05 \%$ and that of guaiacol was $0.075 \mathrm{ml} / 1$. The clearance zone on RBB plates indicates the presence of lignin degrading enzymes which may be lignin peroxidase (LiP), manganese peroxidase $(\mathrm{MnP})$ or laccase. The presence of red zone on guaiacol containing medium indicates the presence of lignin peroxidase and presence of clearance zone indicates either manganese peroxidase or laccase.

\section{Growth Profile of Lignocellulolytic Isolates}

Growth profiles of lignocellulolytic isolates were studied by measuring the colony size 
$\left(\mathrm{cm}^{2}\right)$ on potato dextrose agar medium up to 5 days of incubation period.

\section{Results and Discussion}

Results from Table 1, 2 and 3 showed potency index of isolated cultures (A1 to A34) and standard cultures i.e. Cellulase activity, RBB activity, Guaiacol activity and xylanase activity. The potency index for cellulase activity ranges from 1.05 to 4.71 , forming three different groups i.e. high, moderate and low cellulase producing cultures. The potency index for high cellulase producers ranges from 3.01 to 4.71 which includes isolates no. A4, A5, A7, A9 and A10 among the mesophilic isolates; A21, A25 and A31 among the thermophilc isolates; and Phanaerochaete chrysosporium and Thermoascus aurantiacus from the standard cultures. Phanaerochaete chrysosporium showed maximum cellulase activity with potency index of 4.7. The potency index for moderate cellulase producers ranges from 2.01 to 3.00 including mesophilc isolate $\mathrm{A} 2, \mathrm{~A} 3$ and $\mathrm{A} 8$; thermophilc isolates A16, A17 and A18; standard cultures namely Pleurotus ostratus, Trichoderma reesei, Trichoderma harzianum, Humicola fuscoatra and Penicillium roquefortii. The potency index for low cellulase producers ranges from 1.05 to 2.0. Mesophilic isolates A1, A6, A11, A12, A13, A14; Thermophilic isolates A15, A19, A20, A22, A23, A24, A26, A27, A28, A29, A30, A32, A33, A34; Standard cultures namely Coriolus versicolor and Aspergillus oryzae are included in this category. The potency index for RBB ranges from 0.26 to 2.89. Phanaerochaete chrysosporium showed maximum RBB activity having potency index 2.89 followed by thermophilic isolate A25 (PI = 2.71). For guaiacol activity, many isolates gave negative results. Mesophilc isolate number A10 gave good result. Other isolates which gave positive result includes: A1, A4, A5, A7, A8, A9, A12, A13, A14 (mesophilic); and A20, A21, A25, A31, A34 (thermophilc). Isolate number A2, A3, A6, A11, A15, A16, A17, A18, A19, A22, A23, A24, A26, A27, A28, A29, A30, A32, A33 gave negative result. Among the standard cultures, all standards except Penicillium roquefortii gave positive results on guaiacol containing media i.e. presence of redness zone on guaiacol plates, thus indicating presence of lignin peroxidase.

Results from Table 4, 5 and 6 showed growth profile of all the 14 mesophilc, 20 thermophilc isolates and standard cultures. Maximum colony size of $31.1 \mathrm{~cm}^{2}$ was observed by thermophilc isolate A31 and A28 followed by thermophilc isolate A25 having colony size of $30.6 \mathrm{~cm}^{2}$ while mesophilic isolate A7 showed minimum colony size of $4.1 \mathrm{~cm}^{2}$ on $5^{\text {th }}$ day of incubation.

According to Machado et al., (2005), the potency index of a culture cannot be correlated with the amount of lignocellulose degrading enzymes present. But the intensity of clearance zone may be correlated with the activity of the enzymes. From the literature, many reports can be cited, where potency index is used as criteria for qualitative selection of lignocelluloses degrading cultures (Mtui and Masalu, 2008; Okino et al., 2000). Thirty ligno-cellulolytic fungi from various sources like soil, compost, plant straw and decaying plant twigs were isolated by Barrasa et al., (2009). They identified the isolated fungal cultures on the basis of their morphological characteristics. 
Table.1 Potency index of isolated Mesophilic cultures

\begin{tabular}{|c|c|c|c|c|c|}
\hline \multirow{2}{*}{$\begin{array}{l}\text { Isolate } \\
\text { No. }\end{array}$} & \multirow[t]{2}{*}{ Cultural characteristics } & \multicolumn{4}{|c|}{ Potency index } \\
\hline & & $\begin{array}{l}\text { Cellulase } \\
\text { activity }\end{array}$ & $\begin{array}{l}\text { RBB } \\
\text { activity }\end{array}$ & $\begin{array}{l}\text { Guaiacol } \\
\text { activity }\end{array}$ & $\begin{array}{l}\text { Xylanase } \\
\text { activity }\end{array}$ \\
\hline A1 & $\begin{array}{l}\text { Black biomass, spores, fast growing, no } \\
\text { pigmentation }\end{array}$ & 1.65 & 1.44 & $+\mathrm{ve}$ & 1.60 \\
\hline $\mathrm{A} 2$ & $\begin{array}{l}\text { Greenish black hyphal biomass, fluffy } \\
\text { cottony appearance }\end{array}$ & 2.77 & 0.87 & -ve & 1.98 \\
\hline A3 & $\begin{array}{l}\text { Initially white, then dark greenish hyphal } \\
\text { biomass, fast growing }\end{array}$ & 2.14 & 0.26 & -ve & 1.75 \\
\hline A4 & $\begin{array}{l}\text { Light greenish hyphal biomass, no } \\
\text { pigmentation, fast growing }\end{array}$ & 3.51 & 2.01 & $+\mathrm{ve}$ & 2.16 \\
\hline A5 & $\begin{array}{l}\text { Dark green beautiful colony, spore } \\
\text { forming, fast growing, no pigmentation }\end{array}$ & 3.05 & 1.67 & $+\mathrm{ve}$ & 2.32 \\
\hline A6 & $\begin{array}{l}\text { Light green colored growth, powdery, } \\
\text { small spores }\end{array}$ & 1.51 & 1.56 & -ve & 1.42 \\
\hline A7 & $\begin{array}{l}\text { Black biomass, soft, fast growing, no } \\
\text { pigmentation }\end{array}$ & 3.06 & 1.36 & $+\mathrm{ve}$ & 1.11 \\
\hline A8 & $\begin{array}{l}\text { Brown spores, fast growing, wrinkled } \\
\text { colonies }\end{array}$ & 2.78 & 1.25 & $+\mathrm{ve}$ & 1.44 \\
\hline A9 & $\begin{array}{l}\text { Black spores in centre, whitish ends, axial } \\
\text { look from lower surface }\end{array}$ & 3.02 & 1.56 & $+\mathrm{ve}$ & 1.33 \\
\hline A10 & $\begin{array}{l}\text { White, cottony growth, pale coloured from } \\
\text { lower surface }\end{array}$ & 3.24 & 1.02 & $\begin{array}{l}\text { +ve } \\
\text { (Good) }\end{array}$ & 3.07 \\
\hline A11 & $\begin{array}{l}\text { Dark green, small in size, fast growing, no } \\
\text { pigmentation }\end{array}$ & 1.14 & 1.31 & -ve & 1.44 \\
\hline A12 & $\begin{array}{l}\text { Exact white, pulled up colonies, soft look, } \\
\text { no pigmentation }\end{array}$ & 1.69 & 1.07 & $+\mathrm{ve}$ & 1.14 \\
\hline A13 & $\begin{array}{l}\text { Brown spores, fast growing, no } \\
\text { pigmentation, }\end{array}$ & 1.72 & 1.96 & $+\mathrm{ve}$ & 1.8 \\
\hline A14 & $\begin{array}{l}\text { Black coloured spores, small in size, soft } \\
\text { mat/grassy look }\end{array}$ & 1.89 & 2.04 & $+\mathrm{ve}$ & 2.24 \\
\hline
\end{tabular}

Media used: MEA + Paddy straw (1\%), incubated at $30 \pm 2^{\circ} \mathrm{C}$ for 5-7 days, RBB: Remazol Brilliant Blue (0.05\%), +ve values of RBB plates indicates lignin degradation, -ve value of RBB plates indicates no lignin degradation; Guaiacol i.e. O- methoxy phenol $(0.075 \mathrm{ml} / \mathrm{l})$, +ve/-ve values shows presence/absence of redness zone which indicates the presence of lignin peroxidise 
Table.2 Potency index of isolated Thermophilic cultures

\begin{tabular}{|c|c|c|c|c|c|}
\hline \multirow{2}{*}{$\begin{array}{l}\text { Isolate } \\
\text { No. }\end{array}$} & \multirow[t]{2}{*}{ Cultural characterisitics } & \multicolumn{4}{|c|}{ Potency index } \\
\hline & & $\begin{array}{l}\text { Cellulase } \\
\text { activity }\end{array}$ & $\begin{array}{l}\text { RBB } \\
\text { activity }\end{array}$ & $\begin{array}{l}\text { Guaiacol } \\
\text { activity }\end{array}$ & $\begin{array}{l}\text { Xylanase } \\
\text { activity }\end{array}$ \\
\hline A15 & $\begin{array}{l}\text { Green colonies, soft matty/grassy } \\
\text { look, no pigmentation }\end{array}$ & 1.12 & 1.56 & -ve & 1.51 \\
\hline A16 & $\begin{array}{l}\text { White hyphal biomass, fluffy, no } \\
\text { pigmentation }\end{array}$ & 2.94 & 0.92 & $-\mathrm{ve}$ & 1.84 \\
\hline A17 & $\begin{array}{l}\text { Emarald green, cottony biomass, } \\
\text { filled the plate on } 4^{\text {th }} \text { day }\end{array}$ & 2.56 & 0.69 & -ve & 1.69 \\
\hline A18 & $\begin{array}{l}\text { Black hyphal biomass, small spores, } \\
\text { later on fluffy biomass }\end{array}$ & 2.25 & 0.68 & -ve & 1.14 \\
\hline A19 & $\begin{array}{l}\text { Green colony, soft matty look, no } \\
\text { pigmentation }\end{array}$ & 1.12 & 1.03 & -ve & 1.52 \\
\hline A20 & $\begin{array}{l}\text { Light green colony, powdery, fast } \\
\text { growing }\end{array}$ & 1.56 & + & $+\mathrm{ve}$ & 1.38 \\
\hline A21 & $\begin{array}{l}\text { Black spores, appears as dots of black } \\
\text { colour, fast growing }\end{array}$ & 3.3 & + & $+\mathrm{ve}$ & 1.23 \\
\hline $\mathrm{A} 22$ & $\begin{array}{l}\text { Concentric rings of light and dark } \\
\text { green colour, slow growing, }\end{array}$ & 1.92 & 0.73 & -ve & 1.19 \\
\hline A23 & $\begin{array}{l}\text { Green with maroonish, powdery, no } \\
\text { pigmentation }\end{array}$ & 1.39 & 0.45 & -ve & 1.09 \\
\hline A24 & $\begin{array}{l}\text { Light greenish, clumped colonies, fast } \\
\text { growing }\end{array}$ & 1.2 & 0.68 & -ve & 1.11 \\
\hline $\mathrm{A} 25$ & $\begin{array}{l}\text { Yellowish green, thick mass, no } \\
\text { pigmentation }\end{array}$ & 3.17 & 2.74 & $+\mathrm{ve}$ & 2.25 \\
\hline A26 & $\begin{array}{lll}\begin{array}{l}\text { Light green, wrinkled, no } \\
\text { pigmentation }\end{array} & & \text { no } \\
\end{array}$ & 1.19 & 0.88 & -ve & 1.21 \\
\hline A27 & $\begin{array}{l}\text { Dark green, initially whitish, flat } \\
\text { layer, }\end{array}$ & 1.05 & 0.47 & -ve & 1.02 \\
\hline A28 & $\begin{array}{l}\text { Whitish green, fast growing, no } \\
\text { pigmentation }\end{array}$ & 1.12 & 1.04 & -ve & 1.28 \\
\hline A29 & $\begin{array}{l}\text { Creamish white colony, centre of } \\
\text { colonies pulled up/pointed, no } \\
\text { pigmentation }\end{array}$ & 1.31 & 2.35 & -ve & 1.32 \\
\hline A30 & $\begin{array}{l}\text { Creamish white colony, wrinkled } \\
\text { and clumped, no pigmentation }\end{array}$ & 1.62 & 2.0 & -ve & 1.11 \\
\hline A31 & $\begin{array}{l}\text { Black spores with white ends, soft } \\
\text { growth, axial pattern on lower surface }\end{array}$ & 3.36 & 2.23 & $+\mathrm{ve}$ & 2.06 \\
\hline A32 & $\begin{array}{l}\text { Green, soapy soft powdery, no } \\
\text { pigmentation }\end{array}$ & 1.05 & 1.96 & -ve & 1.87 \\
\hline A33 & $\begin{array}{l}\text { Green small spores, powdery, fast } \\
\text { growing }\end{array}$ & 1.11 & 0.34 & -ve & 1.45 \\
\hline A34 & $\begin{array}{l}\text { Yellowish mat, soft hyphal biomass, } \\
\text { no pigmentation }\end{array}$ & 1.32 & 0.21 & $+\mathrm{ve}$ & 1.16 \\
\hline
\end{tabular}

Media used: MEA + Paddy straw (1\%), incubated at $45 \pm 2^{\circ} \mathrm{C}$ for 5-7 days, RBB: Remazol Brilliant Blue (0.05\%), +ve values of RBB plates indicates lignin degradation, -ve value of RBB plates indicates no lignin degradation; Guaiacol i.e. O- methoxy phenol $(0.075 \mathrm{ml} / \mathrm{l})$, +ve/-ve values shows presence/absence of redness zone which indicates the presence of lignin peroxidise 
Table.3 Potency index of standard cultures

\begin{tabular}{|c|c|c|c|c|c|}
\hline \multirow[t]{2}{*}{ Std. Culture } & \multirow[t]{2}{*}{ Cultural characterisitics } & \multicolumn{4}{|c|}{ Potency index } \\
\hline & & $\begin{array}{l}\text { Cellulase } \\
\text { activity }\end{array}$ & $\begin{array}{l}\text { RBB } \\
\text { activity }\end{array}$ & $\begin{array}{l}\text { Guaiacol } \\
\text { activity }\end{array}$ & $\begin{array}{l}\text { Xylanase } \\
\text { activity }\end{array}$ \\
\hline $\begin{array}{l}\text { Phanaerochaete } \\
\text { chrysosporium } \\
\text { MTCC } 787\end{array}$ & $\begin{array}{l}\text { Whitish, creamy, powdered, no } \\
\text { pigmentation, fast growth }\end{array}$ & 4.71 & 2.89 & $\begin{array}{l}+ \text { ve } \\
\text { (Very } \\
\text { Good) }\end{array}$ & 2.44 \\
\hline $\begin{array}{l}\text { Pleurotus } \\
\text { ostreatus } \\
\text { MTCC } 142\end{array}$ & $\begin{array}{l}\text { Whitish, cottony growth, no } \\
\text { pigmentation }\end{array}$ & 2.83 & 1.07 & $+\mathrm{ve}$ & 1.21 \\
\hline $\begin{array}{l}\text { Coriolus } \\
\text { versicolor } \\
\text { MTCC } 138\end{array}$ & $\begin{array}{l}\text { Creamy whitish, thick mass, no } \\
\text { pigmentation }\end{array}$ & 1.89 & 0.98 & $\begin{array}{l}\text { +ve } \\
\text { (very } \\
\text { good) }\end{array}$ & 2.8 \\
\hline $\begin{array}{l}\text { Trichoderma } \\
\text { reesei } \\
\text { MTCC } 164 \\
\end{array}$ & $\begin{array}{l}\text { Emerald green coloured spores, } \\
\text { fast growing, light yellowish } \\
\text { green in beginning }\end{array}$ & 2.71 & 0.67 & $+\mathrm{ve}$ & 1.61 \\
\hline $\begin{array}{l}\text { Trichoderma } \\
\text { harzianum } \\
\text { MTCC } 792\end{array}$ & $\begin{array}{l}\text { Dark green coloured sporous } \\
\text { growth, radial pattern beneath }\end{array}$ & 2.78 & 1.07 & $+\mathrm{ve}$ & 2.25 \\
\hline $\begin{array}{l}\text { Penicillium } \\
\text { roquefortii } \\
\text { NCIM } 712 \\
\end{array}$ & Green coloured, small spores, & 2.31 & 2.06 & -ve & 1.44 \\
\hline $\begin{array}{l}\text { Aspergillus } \\
\text { oryzae NCIM } \\
1212\end{array}$ & Green colour, powdery growth, & 1.23 & 0.59 & $+\mathrm{ve}$ & 1.69 \\
\hline $\begin{array}{l}\text { Humicola } \\
\text { fuscoatra } \\
\text { MTCC } 1409\end{array}$ & $\begin{array}{l}\text { Light yellowish green, matty } \\
\text { grassy look, }\end{array}$ & 2.12 & 1.1 & $+\mathrm{ve}$ & 1.09 \\
\hline $\begin{array}{l}\text { Thermoascus } \\
\text { aurantiacus } \\
\text { MTCC } 375\end{array}$ & $\begin{array}{l}\text { Soft white, thick cottony biomass, } \\
\text { no pigmentation }\end{array}$ & 3.07 & 2.84 & $+\mathrm{ve}$ & 3.51 \\
\hline
\end{tabular}

Media used: MEA + Paddy straw (1\%), incubated at $30 \pm 2^{\circ} \mathrm{C}$ (mesophilic) and $45 \pm 2^{\circ} \mathrm{C}$ (thermophilic) for 5-7 days, RBB: Remazol Brilliant Blue $(0.05 \%)$, +ve values of RBB plates indicates lignin degradation, -ve value of RBB plates indicates no lignin degradation; Guaiacol i.e. O- methoxy phenol $(0.075 \mathrm{ml} / 1),+v e /-v e$ values shows presence/absence of redness zone which indicates the presence of lignin peroxidise 
Table.4 Growth profile of mesophilic isolates

\begin{tabular}{|c|c|c|c|c|c|c|}
\hline \multirow[t]{2}{*}{ Sr. No. } & \multirow[t]{2}{*}{ Isolate No. } & \multicolumn{5}{|c|}{ Colony size $\left(\mathrm{cm}^{2}\right)$} \\
\hline & & Day 1 & Day 2 & Day3 & Day4 & Day 5 \\
\hline 1. & A1 & 1.4 & 2.8 & 4.3 & 5.6 & 6.9 \\
\hline 2. & $\mathrm{~A} 2$ & 3.4 & 6.6 & 9.4 & 13.2 & 17.7 \\
\hline 3. & A3 & 3.6 & 5.2 & 9.7 & 16.9 & 20.1 \\
\hline 4. & A4 & 4.1 & 8.7 & 13.4 & 17.3 & 21.0 \\
\hline 5. & A5 & 3.5 & 6.1 & 9.8 & 14.3 & 18.9 \\
\hline 6. & A6 & 0.7 & 3.2 & 6.4 & 9.9 & 12.5 \\
\hline 7. & A7 & 0.5 & 1.2 & 2.0 & 2.8 & 4.1 \\
\hline 8. & A8 & 0.3 & 3.4 & 8.7 & 12.8 & 15.7 \\
\hline 9. & A9 & 1.5 & 3.2 & 4.3 & 6.2 & 8.1 \\
\hline 10. & A10 & 0.8 & 3.4 & 7.7 & 10.1 & 14.1 \\
\hline 11. & A11 & 2.4 & 3.8 & 4.9 & 6.8 & 9.6 \\
\hline 12. & A12 & 0.5 & 2.7 & 6.9 & 11.8 & 13.1 \\
\hline 13. & A13 & 1.4 & 3.9 & 7.1 & 10.2 & 13.5 \\
\hline 14. & A14 & Negligible & 1.6 & 4.5 & 8.2 & 13.4 \\
\hline
\end{tabular}

* Media used: Malt Extract Agar with $1 \%$ paddy straw, incubation temperature: $30 \pm 2^{\circ} \mathrm{C}$

Table.5 Growth profile of Thermophilic isolates

\begin{tabular}{|l|l|l|l|l|l|l|}
\hline \multirow{2}{*}{ Sr. No. } & \multirow{2}{*}{ Isolate No. } & \multicolumn{5}{|c|}{ Colony size $\left(\mathrm{cm}^{2}\right)$} \\
\cline { 3 - 7 } & & \multicolumn{1}{|c|}{ Day 1 } & \multicolumn{1}{|c|}{ Day 2 } & Day3 & \multicolumn{1}{c|}{ Day4 } & Day 5 \\
\hline 1. & A15 & 0.7 & 5.8 & 9.1 & 13.4 & 16.2 \\
\hline 2. & A16 & 5.2 & 6.5 & 9.2 & 12.4 & 15.8 \\
\hline 3. & A17 & 5.8 & 7.1 & 10.7 & 14.1 & 17.7 \\
\hline 4. & A18 & 5.6 & 8.3 & 11.9 & 15.2 & 19.3 \\
\hline 5. & A19 & 1.1 & 5.4 & 9.8 & 13.5 & 17.1 \\
\hline 6. & A20 & 0.8 & 4.1 & 8.6 & 14.2 & 19.1 \\
\hline 7. & A21 & 1.7 & 4.2 & 7.9 & 12.6 & 16.7 \\
\hline 8. & A22 & 0.8 & 6.4 & 11.7 & 21.7 & 24.7 \\
\hline 9. & A23 & 0.5 & 1.4 & 3.4 & 8.1 & 11.2 \\
\hline 10. & A24 & 1.0 & 3.5 & 10.7 & 19.9 & 22.1 \\
\hline 11. & A25 & 0.8 & 3.5 & 11.1 & 19.8 & 30.6 \\
\hline 12. & A26 & 0.4 & 3.4 & 12.7 & 25.8 & 33.7 \\
\hline 13. & A27 & 0.4 & 8.1 & 15.2 & 23.1 & 30.3 \\
\hline 14. & A28 & 0.3 & 3.2 & 11.3 & 26.2 & 31.1 \\
\hline 15. & A29 & 0.7 & 4.6 & 8.1 & 12.5 & 16.3 \\
\hline 16. & A30 & 1.4 & 4.2 & 6.5 & 8.1 & 10.2 \\
\hline 17. & A31 & 1.2 & 3.2 & 11.3 & 26.2 & 31.1 \\
\hline 18. & A32 & 0.2 & 2.4 & 4.7 & 7.1 & 9.3 \\
\hline 19. & A33 & 0.4 & 3.3 & 6.9 & 10.4 & 14.1 \\
\hline 20. & A34 & 1.2 & 3.6 & 7.2 & 10.2 & 12.4 \\
\hline
\end{tabular}

* Media used: Malt Extract Agar with $1 \%$ paddy straw, incubation temperature: $45 \pm 2^{\circ} \mathrm{C}$ 
Table.6 Growth profile of Standard Cultures

\begin{tabular}{|c|c|c|c|c|c|c|}
\hline Sr. No. & Std. Cultures & \multicolumn{5}{|c|}{ Colony size $\left(\mathrm{cm}^{2}\right)$} \\
\hline \multicolumn{2}{|c|}{ Mesophilic Cultures: } & Day 1 & Day 2 & Day3 & Day4 & Day 5 \\
\hline 1. & $\begin{array}{l}\text { P. chrysosporium MTCC } \\
787\end{array}$ & 3.2 & 6.4 & 10.1 & 13.7 & 19.4 \\
\hline 2. & P. ostreatus MTCC 142 & 0.5 & 2.5 & 4.1 & 11.1 & 13.2 \\
\hline 3. & C. versicolor MTCC 138 & 0.3 & 2.2 & 3.5 & 6.2 & 7.1 \\
\hline 4. & T. reesei MTCC 164 & 2.0 & 2.8 & 3.9 & 4.8 & 5.5 \\
\hline 5. & T. harzianum MTCC 792 & 1.0 & 2.1 & 3.4 & 4.7 & 5.9 \\
\hline 6. & P. roquefortii NCIM 712 & 0.4 & 2.9 & 4.6 & 7.3 & 10.1 \\
\hline 7. & A. oryzae NCIM 1212 & 1.2 & 2.5 & 3.8 & 5.1 & 7.2 \\
\hline \multicolumn{7}{|c|}{ Thermophilic Cultures: } \\
\hline 8. & H. fuscoatra MTCC 1409 & 0.8 & 2.5 & 5.3 & 7.6 & 10.5 \\
\hline 9. & T. aurantiacus MTCC 375 & 0.4 & 2.3 & 3.9 & 5.8 & 9.1 \\
\hline
\end{tabular}

* Media used: Malt Extract Agar with $1 \%$ paddy straw, incubation temperature: $30 \pm 2^{\circ} \mathrm{C}$ (mesophilic) and $45 \pm 2^{\circ} \mathrm{C}$ (thermophilic).

The isolated cultures were compared for their ability to decolorize reactive black 5 and reactive blue 38 at concentration of 75 and $150 \mathrm{mg} / \mathrm{L}$ respectively to evaluate lignolytic activity. Aspergillus $\mathrm{s} p$, Paecilomyces $s p$ and Sporotrichum $s p$ were identified as most efficient lignocellulose degraders by Mandhulika et al., (1993). Remazol Brilliant Blue R (RBBR) dye was used as a substrate to evaluate lignolytic activity in 125 basidiomycetes fungi isolated from tropical ecosystem and higher level of peroxidase and laccase could not be related to high RBBR decolorization, though intensity of decolorization was in correlation to enzyme production (Machado et al., 2005). Sadaf et al., (2005) reported that majority of Aspergillus and Penicillium sps. were found to posses cellulolytic activity.

From the above studies it is concluded that the mesophilic isolate numbers A4 and A5; thermophilc isolates A25 and A31; and standard cultures namely Phanaerochaete chrysosporium and Thermoascus aurantiacus are the potential lignocellulolytic cultures.

\section{Acknowledgment}

This work has been financially supported by All India Coordinated Research Project on Renewable Energy (AICRP) funding agency.

\section{References}

Barbosa, O., Torres, R., Ortiz, C., Berenguer-Murcia, A., Rodrigues, R.C., Fernandez-Lafuente, R.2013. Heterofunctional Supports in Enzyme Immobilization: From Traditional Immobilization Protocols to Opportunities in Tuning Enzyme Properties. Biomacromolecules, 14: 2433-2462.

Barrasa, J.M., Martinez, A.T. and Martinez, M.J. 2009. Isolation and selection of 
novel basidiomycetes for decolorization of recalcitrant dyes. Folia Microbiol., 54: 59-66.

Bharagava, R.N., Chandra, R., Rai, V. 2009. Isolation and characterization of aerobic bacteria capable of the degradation of synthetic and natural melanoidins from distillery effluent. World J. Microbiol. Biotechnol., 25:737-744

Conesa, A., van den Hondel, C., Punt, P. 2000. Studies on the production of fungal peroxidases in Aspergillus niger. Appl. Environ. Microbiol., 66:3016-3023

Dashtban, M., Schraft, H., Qin, W. 2009. Fungal Bioconversion of Lignocellulosic Residues; Opportunities \& Perspectives. Int. J. Biol. Sci., 5: 578-595.

Dewan, S. 2012. Enzymes in Industrial Applications: Global Markets. Report BIO030G, BCC Research, Wellesley, MD.

Ferreira, H. M. F., Filho, E.X.F. 2004. Purification and characterization of a $\square$-mannanase from Trichoderma harzianum starin T4. Carbohydrate Polymers, 57: 23-29.

Hakala, T., Hildén, K., Maijala, P., Olsson, C., Hatakka, A. 2006. Differential regulation of manganese peroxidases and characterization of two variable $\mathrm{MnP}$ encoding genes in the white-rot fungus Physisporinus rivulosus. Appl. Microbiol. Biotechnol., 73:839-849

Heck, J.X., de-Barros-Soares, L.H., Ayub, M.A.Z. 2005. Optimization of xylanase and mannanase production by Bacillus circulans Strain BL53 on solid state fermentation. Enzyme Microbial Technol., 37: 417-423.

Hofrichter, M. 2002. Review: lignin conversion by manganese peroxidase (MnP). Enzyme Microbial Technol., 30: 454-466.
Irie, T., Honda, Y., Watanabe, T., Kuwahara, M. 2001. Homologous expression of recombinant manganese peroxidase genes in ligninolytic fungus Pleurotus ostreatus. Appl. Microbiol. Biotechnol., 55: 566-570.

Jiang, F., Kongsaeree, P., Schilke, K., Lajoie, C., Kelly, C. 2008a. Effects of $\mathrm{pH}$ and temperature on recombinant manganese peroxidase production and stability. Appl. Biochem. Biotechnol., 146:15-27

Jiang, Z., Wei, Y., Li, D., Li, L., Chai, P., Kusakabe, I. 2006. High level production, purification and characterization of a thermostable $\square$ mannanase from a newly isolated Bacillus subtilis WY34. Carbohydrate Polymers, 66: 88-96.

Jimenez-Tobon, G., Kurzatkowski, W., Rozbicka, B., Solecka, J., Pocsi, I., Penninckx, M. 2003. In situ localization of manganese peroxidase production in mycelia pellets of Phanerochaete chrysosporium. Microbiology-Sgm, 149:3121-3127.

Johnson, T., Pease, E.A., Li, J., Tien, M. 1992. Production and characterization of recombinant lignin peroxidase isozyme $\mathrm{H} 2$ from Phanerochaete chrysosporium using recombinant baculovirus. Arc. Biochem. Biophys., 296(2):660-666

Jørgensen, H. 2003. Production and characterization of cellulases and hemicellulases produced by Penicillium strains. Denmark: Technical University of Denmark. (Ph.D. Thesis within Center for Process Biotechnology, BioCentrumDTU).

Jung, Y.H., Kim, I.J.,Han, J.-I., Choi, I.G.,Kim, K.H. 2011. Aqueous Ammonia Pretreatment of Oil Palm Empty Fruit Bunches for Ethanol Production. Bioresource Technol., 
102:9806-9809.

Kamitsuji, H., Honda, Y., Watanabe, T., Kuwahara, M. 2004. Production and induction of manganese peroxidase isozymes in a white-rot fungus Pleurotus ostreatus. Appl. Microbiol. Biotechnol., 65: 287-294

Kang, S., Park, Y., Lee, J., Hong, S., Kim, S. 2004. Production of Cellulases and Hemicellulases by Aspergillus niger KK2 From Lignocellulosic Biomass. Bioresource Technol., 91: 153-156.

Krisana, A., Rutchadaporn, S., Jarupan, G., Lily, E., Sutipa, T., Kanyawim, K. 2005. Endo-1,4- $\beta$-xylanase from Aspergillus cf. niger BCC14405 isolated in Thailand: purification, characterization andgene isolation. $J$. Biochem. Mol. Biol., 38:17-23.

Lankinen, P., Hilden, K., Aro, N., SalkinojaSalonen, M., Hatakka, A. 2005. Manganese peroxidase of Agaricus bisporus: grain bran-promoted production and gene characterization. Appl. Microbiol. Biotechnol, 66:401407

Li, D., Youngs H, Gold M (2001) Heterologous expression of a thermostable manganese peroxidase from Dichomitus squalens in Phanerochaete chrysosporium. Arch. Biochem. Biophys., 385:348-356

Ljungdahl, L.G. 2008. The cellulase/hemicellulase system of the anaerobic fungus Orpinomyces PC-2 and aspects of its applied use. Ann NY Acad Sci., 1125: 308-321.

Lynd, L.R., Weimer P.J., van Zyl W.H., and Pretorius I.S. 2002. Microbial Cellulose Utilization: Fundamentals and Biotechnology. Microbiol. Mol. Biol. Reviews, 66: 506-577.

Machado, K.G.M., Matheus, D.R. and Bononi, V.L.R. 2005. Ligninolytic enzyme production and remazol brilliant blue $\mathrm{R}$ decolorization by tropical brazillian basidiomycetes. Braz. J. Microbiol., 36: 246-52.

Mandhulika, Singh, D.P. and Malik, R.K. 1993. Isolation of a few lignocelluloses degrading fungi. Ind. $J$. Microbiol., 33: 265-67.

Mishra, M., Thakur, I. 2010. Isolation and characterization of alkalotolerant bacteria and optimization of process parameters for decolorization and detoxification of pulp and paper mill effluent by Taguchi approach. Biodegradation, 21:967-978

Mitchell, D.A., Berovic, M., Krieger, N. 2006. Solid-State Fermentation Bioreactor Fundamentals: Introduction and Overview. Springer, New York.

Mtui, G. and Masalu, R. 2008. Extracellular enzymes from brown rot fungus Laetiporus sulphureus isolated from mangrove forests of coastal Tanzania. Scientific Research and Essay, Academic J., 3: 154-61.

Nagendran, S., Hallen-Adams, H.E., Paper, J.M., Aslam, N., Walton, J.D. 2009. Reduced genomic potential for secreted plant cell-wall-degrading enzymes in the ectomycorrhizal fungus Amanita bisporigera, based on the secretome of Trichoderma reesei. Fungal genet. Biol., 46: 427-435.

Nuske, J., Scheibner, K., Dornberger, U., Ullrich, R., Hofrichter, M. 2002. Large scale production of manganeseperoxidase using agaric white-rot fungi. Enzyme Microb. Technol., 30:556-561

Okino, L.K., Machado, K.G.M., Fabric, C. and Bonomi, V.L.R. 2000. Ligninolytic activity of tropical rainforest basidiomycetes. World $J$. Microbiol. Biotechnol., 16: 889-93.

Pal, A., Khanum, F. 2010. Production and Extraction Optimization of Xylanase From Aspergillus niger DFR-5 Through Solid-State-Fermentation. 
Bioresource Technol., 101:7563-7569. Palma, C., Martínez, A., Lema, J., Martínez, M. 2000. Different fungal manganeseoxidizing peroxidases: a comparison between Bjerkandera sp. And Phanerochaete chry-sosporium. $J$. Biotechnol., 77:235-245

Pease, E., Aust, S., Tien, M. 1991. Heterologous expression of active manganese peroxidase from Phanerochaete chrysosporium using the baculovirus expression system. Biochem. Biophys. Research. Comm., 179:897-903

Peciulyt, D. 2007. Isolation of cellulolytic fungi from waste paper gradual recycling materials. Ekologija, 53: 1118.

Petruccioli, M., Frasconi, M., Quaratino, D., Covino, S., Favero, G., Mazzei, F., Federici, F., D’Annibale, A. 2009. Kinetic and redox properties of $\mathrm{MnP}$ II, a major manganese peroxidase isoenzyme from Panus tigrinus CBS 577.79. J. Biol. Inorg. Chem., 14:1153-1163

Prathumpai, W. 2003. Enzyme production in Aspergilli on different carbon sources. Denmark: Technical University of Denmark. (Ph.D. Thesis within Center for Process Biotechnology, BioCentrum-DTU).

Sadaf, J., Khan, N., Jahangeer, S., Sohali, M., Shahzad, S., Ahmad, A., Khan, S.A. 2005. Screening and characterization of fungal cellulases isolated from the native environmental source. Pak. J. Botany, 37(3): 739748.

Sanchez, C. 2009. Lignocellulosic residues: biodegradation and bioconversion by fungi. Biotechnol. Adv., 27: 185-194.

Schmidt, O. 2006. Wood and Tree Fungi: Biology, Damage, Protection, and Use, Chapter 3: Physiology and Chapter 4: Wood cell wall degradation. Berlin; New York: Springer.

Silva, E., Martins, S., Milagres, A. 2008. Extraction of manganese peroxidise produced by Lentinula edodes. Biores. Technol., 99:2471-2475

Singh, D., Zeng, J., Chen, S. 2011. Increasing manganese peroxidase productivity of Phanerochaete chrysosporium by optimizing carbon sources and supplementing small molecules. Lett. Appl. Microbiol., 53:120-123

Sklenar, J., Niku-Paavola, M.L., Santos, S., Man, P., Kruus, K., Novotny, C. 2010. Isolation and characterization of novel pI 4.8 MnP isoenzyme from white-rot fungus Irpex lacteus. Enzyme Microb. Technol., 46:550-556

Steffen, K., Hofrichter, M., Hatakka, A. 2002. Purification and characterization of manganese peroxidases from the litter-decomposing basidiomycetes Agrocybe praecox and Stropharia coronilla. Enzyme Microb. Technol., 30:550-555

Susla, M., Novotny, C., Erbanova, P., Svobodova, K. 2008. Implication of Dichomitus squalens manganesedependent peroxidase in Dye decolorization and cooperation of the enzyme with laccase. Folia Microbiol., 53:479-485

Taboada-Puig, R., Lú-Chau, T., Moreira, M., Feijoo, G., Martínez, M., Lema, J. 2011. A new strain of Bjerkandera sp. production, purification and characterization of versatile peroxidase. World J. Microbiol. Biotechnol., 27:115-122

Tamaru, Y., Araki, T., Amagoi, H., Mori, H., Morishita, T. 1995. Purification and characterization of an extracellular $\square$-1,4-mannanase from a marine bacterium Vibrio sp. Strain MA-138. Applied and Environmental 
Microbiol., 61: 4454-4458.

Wang, P., Hu, X., Cook, S., Begonia, M., Lee, K., Hwang, H.M. 2008. Effect of culture conditions on the production of ligninolytic enzymes by white rot fungi Phanerochaete chrysosporium (ATCC 20696) and separation of its lignin peroxidase. World J. Microbiol. Biotechnol., 24:2205-2212

Weng, J.K., Li, X., Bonawitz, N.D., Chapple, C. 2008. Emerging strategies of lignin engineering and degradation for cellulosic biofuel production. Curr. Opin. Biotechnol., 19: 166-172.

Whitwam, R., Tien, M. 1996. Heterologous expression and reconstitution of fungal
Mn peroxidase. Arch. Biochem. Biophys., 333:439-446

Yadav, S., Chandra, R., Rai, V. 2011. Characterization of potential MnP producing bacteria and its metabolic products during decolourisation of synthetic melanoidins due to biostimulatory effect of D-xylose at stationary phase. Process Biochem., 46(9):1774-1784

Yoon, J.J., Cha, C.J., Kim, Y.S., Son, D.W., Kim, Y.K. 2007. The brown-rot basidiomycete Fomitopsis palustris has the endo-glucanases capable of degrading microcrystalline cellulose. J. Microbiol, Biotechnol., 17: 800-805.

\section{How to cite this article:}

Ajit Kaur and Urmila Gupta Phutela. 2016. Isolation and Qualitative Selection of Fungi for Production of Lignocellulolytic Enzymes. Int.J.Curr.Microbiol.App.Sci. 5(6): 718-730. doi: http://dx.doi.org/10.20546/ijcmas.2016.506.078 